\title{
Utility of blue-on-yellow perimetry in the evaluation of patients with idiopathic optic neuropathy
}

This article was published in the following Dove Press journal:

Clinical Ophthalmology

27 June 2013

Number of times this article has been viewed

\section{Shinji Makino}

Department of Ophthalmology, Jichi Medical University, Shimotsuke, Tochigi, Japan
Correspondence: Shinji Makino

33 II-I Yakushiji, Shimotsuke,

Tochigi 329-0498, Japan

Tel +8I 285587382

Fax +8I 285448365

Email makichan@jichi.ac.jp
Abstract: A 68-year-old woman with bilateral idiopathic optic neuropathy was examined using blue-on-yellow perimetry. Best-corrected visual acuity was 0.5 and 0.6 in her right and left eyes, respectively. Visual field testing with a Goldmann perimeter showed constriction of the I/2 e isopter in both eyes. With regard to mean deviation, the results $(-13.18 \mathrm{~dB}$ right, $-10.01 \mathrm{~dB}$ left $)$ of blue-on-yellow perimetry were worse than those obtained using white-on-white perimetry $(-1.26 \mathrm{~dB}$ right, $-1.83 \mathrm{~dB}$ left). Optical coherence tomography revealed decreased retinal nerve fiber layer thickness in both eyes. Blue-on-yellow perimetry is useful for the detection of visual field disturbances in patients with optic neuropathy.

Keywords: optic neuropathy, blue-on-yellow perimetry, white-on-white perimetry, optical coherence tomography, retinal thickness.

\section{Introduction}

There are three types of cones in the human retina, with differing sensitivity to long, medium, and short wavelengths. Blue-on-yellow perimetry, or short-wavelength automated perimetry, has gained popularity because of utility in the early detection of glaucomatous visual field loss. This approach to perimetry is designed to evaluate the function of the cones most sensitive to short-wavelength light. A bright yellow background hyperpolarizes the rods as well as cones sensitive to middle- and longwavelength light, so that only the cones that respond to short-wavelength light are able to detect the target. ${ }^{1}$ Numerous studies on patients with ocular hypertension or early-stage glaucoma have suggested that defects detectable by blue-on-yellow perimetry can be recognized earlier than the defects that are detectable with white-on-white perimetry. ${ }^{1-6}$ Furthermore, longitudinal studies of patients with glaucoma have shown that blue-onyellow visual field defects may progress faster than white-on-white defects. ${ }^{1-6}$

Short-wavelength stimuli have been used for many years to detect abnormalities in the retina and optic nerve. ${ }^{1}$ The utility of blue-on-yellow perimetry has been discussed in connection with other retinal diseases such as age-related macular disease, night blindness, and idiopathic blind spot enlargement syndrome. ${ }^{1,7-9}$ To my knowledge, few reports have investigated the utility of blue-on-yellow perimetry in the treatment of neuro-ophthalmological disorders. ${ }^{1,10-14}$ Herein, I report the value of blue-on-yellow perimetry in a patient with idiopathic optic neuropathy.

\section{Case report}

A 68-year-old woman was referred to the Jichi Medical University Hospital due to visual disturbances that began several years ago. Her personal and family history and 
systemic evaluation were unremarkable. On ophthalmic examination, the best-corrected visual acuity (BCVA) in the right eye was 0.5 with $-0.50 \mathrm{D}-2.50 \mathrm{D} \times 90^{\circ}$; $\mathrm{BCVA}$ in the left eye was 0.6 with $+0.50 \mathrm{D}-1.75 \mathrm{D} \times 105^{\circ}$. The patient was orthophoric and showed normal ocular movements. On slit-lamp examination, mild cortical opacities were detected in both lenses. Ocular pressures were normal. The fundus examination showed diffuse optic disc pallor in both eyes (Figure 1A and B). Visual field testing with a Goldmann perimeter showed constriction of the $\mathrm{I} / 2 \mathrm{e}$ isopter in both eyes (Figure 2A and B). White-on-white perimetry and blueon-yellow perimetry were performed using program 24-2 (FASTPAC) of a Humphrey Field Analyzer (model 750; Humphrey Instruments, Inc, Dublin, CA, USA). All testing was performed with an appropriate near add and in adherence with the instructions provided in the Humphrey perimetry manual. All white-on-white perimetry was performed with a $10 \mathrm{~cd} / \mathrm{m}^{2}$ white background and a size III white stimulus. Blue-on-yellow perimetry was performed with a $100 \mathrm{~cd} / \mathrm{m}^{2}$ yellow background and a size V blue $(440 \mathrm{~nm})$ stimulus. All the visual fields included in the data analysis had acceptable visual field reliability indices (fixation loss $<20 \%$, falsepositive or false-negative responses $<33 \%$ ). The results of blue-on-yellow perimetry were less precise than those obtained using white-on-white perimetry $(-13.18 \mathrm{~dB}$ right, $-10.01 \mathrm{~dB}$ left versus $-1.26 \mathrm{~dB}$ right, $-1.83 \mathrm{~dB}$ left) (Figure 3 ). The pattern standard deviation was also worse with blue-on-yellow perimetry versus white-on-white perimetry (3.89 dB right, $4.03 \mathrm{~dB}$ left versus $1.78 \mathrm{~dB}$ right, $1.76 \mathrm{~dB}$ left) (Figure 3). Color vision was normal when tested using Ishihara color plates. The patient also passed the Farnsworth dichotomous test. Optical coherence tomography (OCT) (RS-3000; NIDEK, Gamagori, Japan) was used to measure retinal nerve fiber layer (RNFL) thickness in a circular area centered on the optic disc. RNFL thickness measurements were averaged for each quadrant of this circle and for the circular area overall. OCT was also used to map
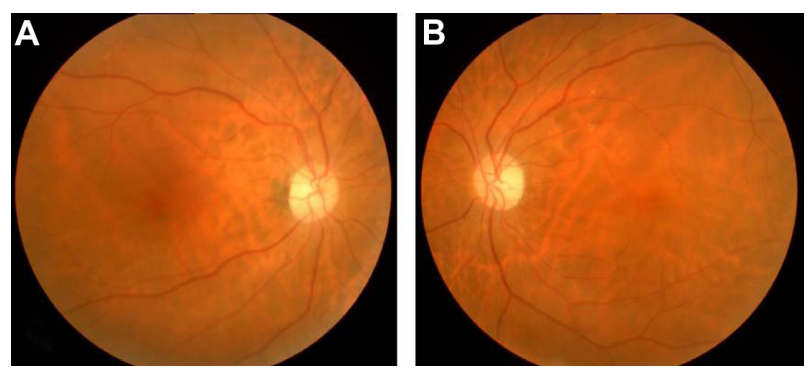

Figure I Right (A) and left (B) fundus photographs showing diffuse optic disc pallor in both eyes. the RNFL, ganglion cell layer (GCL), and inner plexiform layer (IPL). The latter two maps were overlapped to measure retinal thickness at the macula. Measurements were obtained at an inner ring (1.5-4.5 mm from the central fovea) and at an outer ring (4.5-9.0 $\mathrm{mm}$ from the central fovea). No measurement was obtained at the central fovea. The results showed decreased retinal thickness at each measurement point (Figure 4). The decrease was much more marked in the combined NFL, GCL, and IPL map as compared with the RNFL map. In the right eye, mean RNFL thickness at the temporal, superior, nasal, and inferior quadrants was 47, 93, 60 , and $75 \mu \mathrm{m}$, respectively. In the left eye, these values were 47, 110, 41, and $96 \mu \mathrm{m}$, respectively (Figure 4A and D). The combined NFL, GCL, and IPL map showed that thickness at the superior and inferior segments was 64 and $59 \mu \mathrm{m}$, respectively, in the right eye. In the left eye, these values were 65 and $62 \mu \mathrm{m}$, respectively. In addition, combined NFL, GCL, and IPL map revealed that the thickness at the superotemporal, inferotemporal, superonasal, and inferonasal quadrants were $65,59,67$, and $57 \mu \mathrm{m}$, respectively at the inner ring in the right eye. In the left eye, these values were $67,59,70$, and $70 \mu \mathrm{m}$, respectively. Measurements at the outer ring, in the right eye, were as follows: 57, 54, 72, and $66 \mu \mathrm{m}$, respectively. In the left eye, these values were 58, 54, 71, and $69 \mu \mathrm{m}$, respectively (Figure 4B, C, E, and F). Comparison with the normative database showed that the combined thickness of the NFL, GCL, and IPL as measured at the inner ring was decreased in comparison with $>99 \%$ of the population in both eyes.

\section{Discussion}

Few reports in the literature have used blue-on-yellow perimetry to investigate the functional manifestations of neuro-ophthalmological disorders. ${ }^{10-14}$ In a report by Keltner and Johnson, ${ }^{10}$ one patient exhibited hemianopsia on blueon-yellow perimetry despite a normal performance on standard automated perimetry. The authors evaluated patients with optic neuritis, multiple sclerosis, and other neuroophthalmologic disorders using blue-on-yellow perimetry. This approach often revealed more extensive visual field loss than was observed using standard automated perimetry. Wakai et $\mathrm{al}^{11}$ highlighted the utility of blue-on-yellow perimetry in monitoring recovery from optic neuropathy. Their study showed differences between blue-on-yellow and white-on-white perimetry with respect to both the level of visual function as well as the time courses of these changes. They confirmed that blue-on-yellow perimetry evaluated visual field deficits distinct from those evaluated 

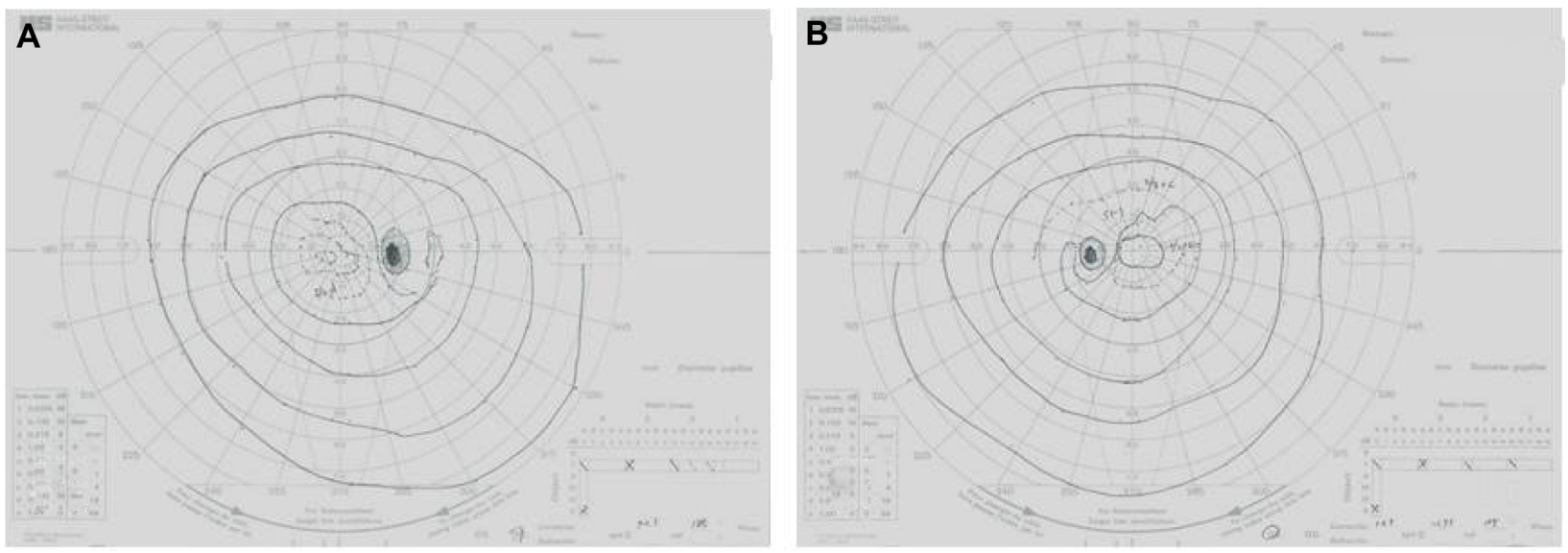

Figure 2 Right (A) and left (B) Goldmann perimetry showed constriction of the $1 / 2$ e isopter in both eyes.

by white-on-white perimetry. The discrepancy stems from the former's use of a mechanism sensitive to short wavelengths. Walters et $\mathrm{al}^{12}$ evaluated short wavelength-automated perimetry in autosomal dominant optic neuropathy. The results suggested that short wavelength-automated perimetry might be a robust indicator in both early and late stages of this disease. Fujimoto et a $\mathrm{l}^{13,14}$ reported on their study of multiple sclerosis patients with quadrantanopia and/or sectoranopia. Blue-on-yellow perimetry is useful in uncovering visual field defects that go undetected by conventional white-on-white perimetry.

Machida et $\mathrm{al}^{9}$ reported on their evaluation of a patient with acute idiopathic blind-spot enlargement syndrome who was evaluated using both white-on-white and blueon-yellow perimetry. The difference in the mean deviation was significantly greater for blue-on-yellow perimetry as compared with white-on-white perimetry. The authors recommend blue-on-yellow perimetry as a sensitive measure
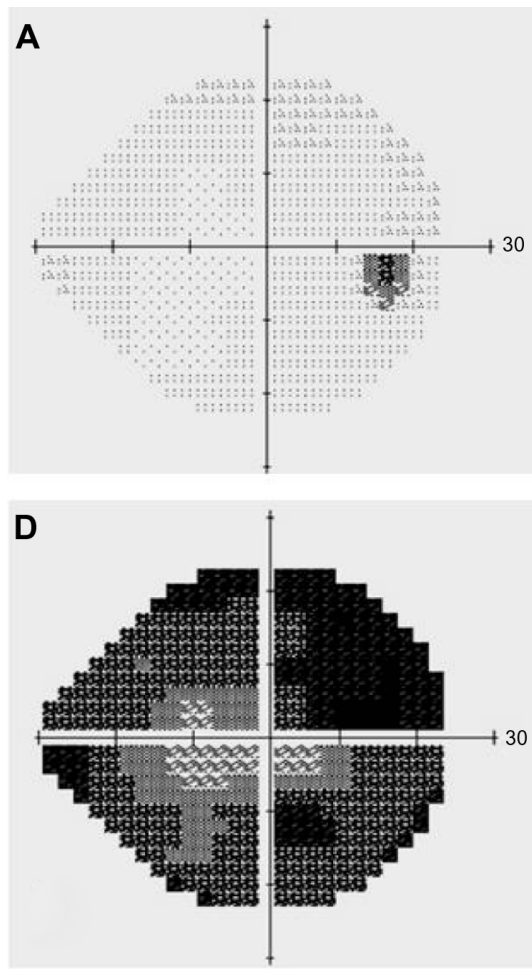
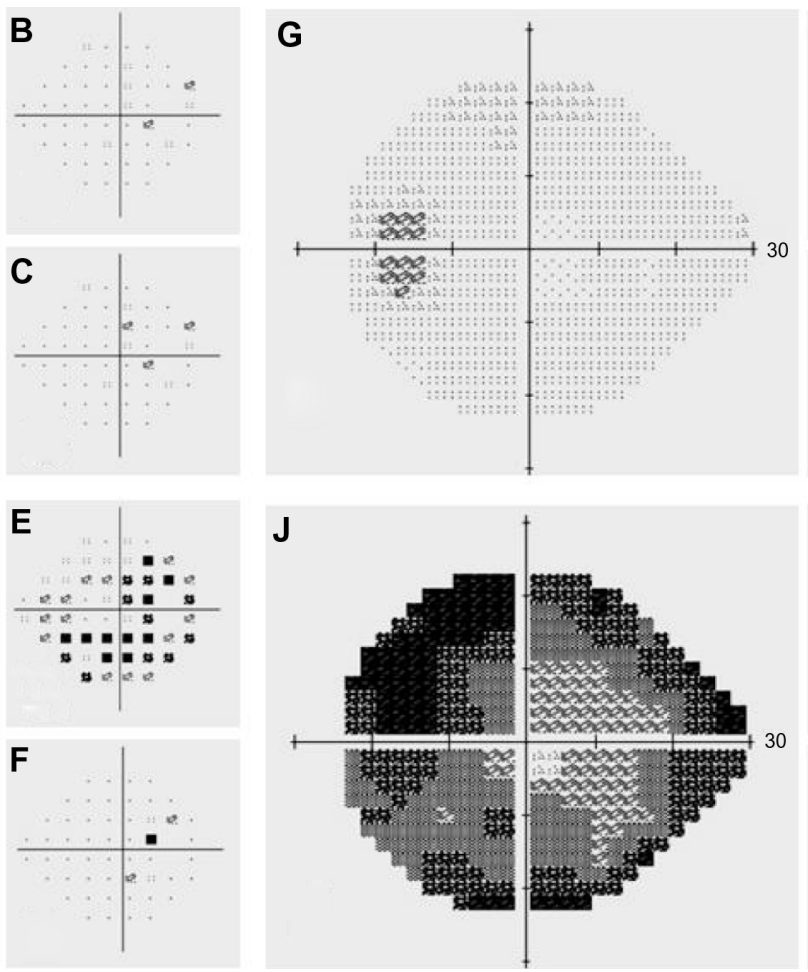
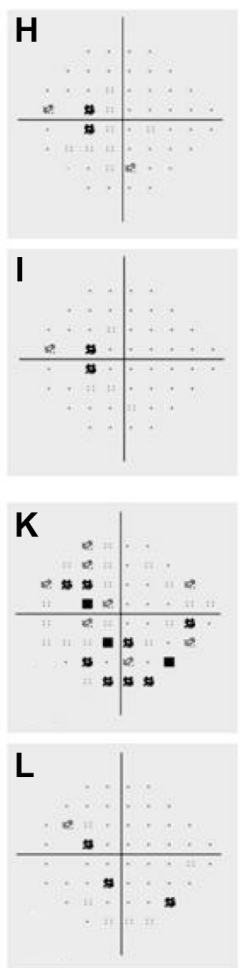

Figure 3 Results of right (A-F) and left (G-L) white-on-white perimetry (A-C and $\mathbf{G}-\mathbf{I})$ and blue-on-yellow perimetry (D-F and J-L). Gray-tone scale (A, D, G, and J), total deviation map (B, E, H, and $\mathbf{K}$ ) and pattern standard deviation map (C, F, I, and $\mathbf{L}$ ) for white-on-white and blue-on-yellow perimetry. Note that blue-on-yellow perimetry captured the visual field abnormalities more effectively than white-on-white perimetry. 


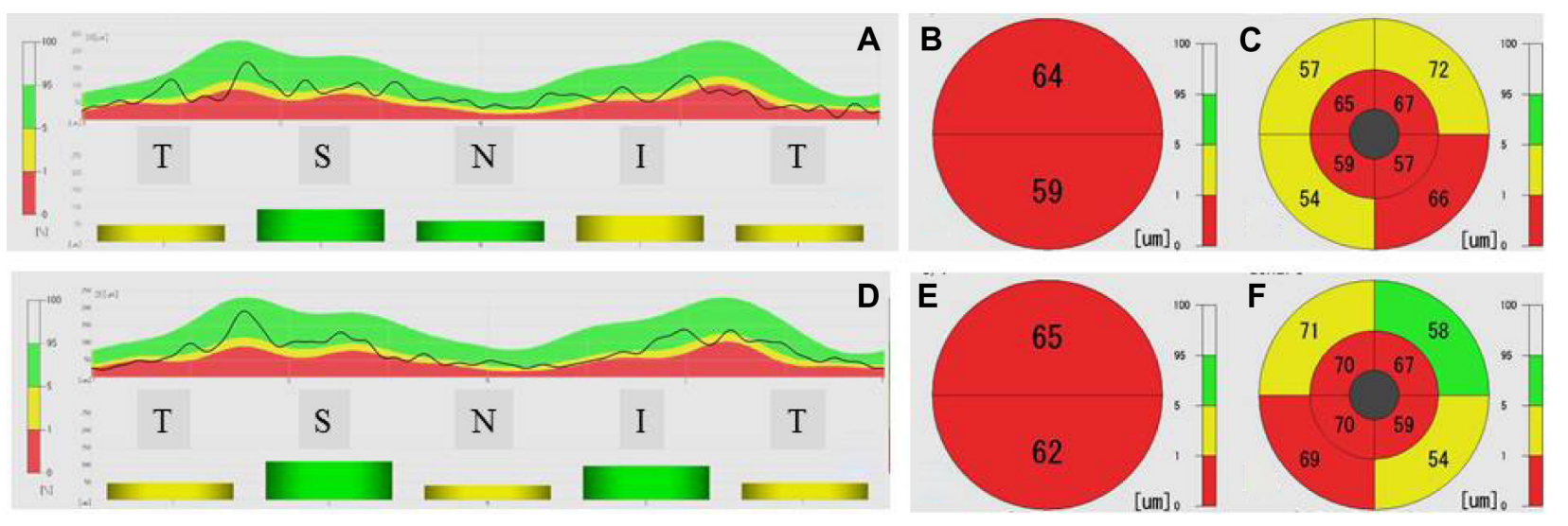

Figure 4 Right (A-C) and left (D-F) optical coherence tomography scans showing decreased retinal thickness.

Notes: A and D: RNFL map, B and E: RNFL + GCL + IPL map $(9.0 \mathrm{~mm}), \mathrm{C}$ and F: RNFL + GCL + IPL map (I.5/4.5/9.0 mm).

Abbreviations: RNFL, retinal nerve fiber layer; GCL, ganglion cell layer; IPL, inner plexiform layer; T, temporal; S, superior; N, nasal; I, inferior.

with which to detect retinal dysfunction that is not detected by white-on-white perimetry.

Two possible explanations can be considered for why blue-cone sensitivity is more vulnerable to pathological processes in comparison with other cone systems. First, blue cones may be more delicate physiologically and more susceptible to photoreceptor diseases. ${ }^{9}$ Second, the response range over which the blue cone functions is significantly limited compared with those of the other cones. Because of this difference, an equivalent loss of response in each cone produces a larger elevation of the threshold in the blue cone system. ${ }^{9}$

Two factors should be considered for evaluating the results of blue-on-yellow perimetry. The first is the influence of cataract. Kim et a ${ }^{15}$ demonstrated improvements in mean deviation after cataract surgery in blue-on-yellow as well as white-on-white perimetry. However, the mean deviation change in blue-on-yellow perimetry was far greater than the mean deviation change in white-on-white perimetry. These findings show that cataracts affect visual function as measured by both blue-on-yellow and white-on-white perimetry, but the reduction in blue-on-yellow sensitivity is far greater than that of white-on-white sensitivity.

Although the mean deviation index should be used with great caution when evaluating a patient using blue-on-yellow perimetry, the lens opacities observed in this case did not have a significant effect on the patient's vision. The second factor to consider is result reproducibility over time. Short- and long-term fluctuations in the visual fields of normal subjects were greater when measured by blue-on-yellow perimetry versus white-on-white perimetry. ${ }^{16}$

With respect to the OCT findings, Ito et $\mathrm{al}^{17}$ found that RNFL thickness in the macular area was significantly reduced in patients with autosomal dominant optic neuropathy as compared with normal subjects. This trend was true at all measured data-points, especially at $1 \mathrm{~mm}$ from the fovea. Combined thickness of the GCL, IPL, inner nuclear layer, and outer plexiform layer as measured in the macular area were significantly reduced compared with the values measured in normal patients. Takasugi et al ${ }^{18}$ also demonstrated atrophy of the peripapillary RNFL in autosomal dominant optic neuropathy.

In conclusion, the above results showed that blue cone sensitivity is diffusely depressed in eyes with optic neuropathy. Furthermore, blue-on-yellow perimetry represents a sensitive method with which to detect retinal dysfunction that is not detected by white-on-white perimetry. Finally, the conclusions that can be drawn from these findings are limited due to the nature of the study. The reader must consider that these findings were based on a single case of idiopathic optic neuropathy. Additional studies with additional cases will be necessary.

\section{Disclosure}

There are no conflicts of interest in relation to this paper.

\section{References}

1. Sample PA. Short-wavelength automated perimetry: it's role in the clinic and for understanding ganglion cell function. Progr Retin Eye Res. 2000;19:369-383.

2. Teesalu P, Airaksinen PJ, Tuulonen A. Blue-on-yellow visual field and retinal nerve fiber layer in ocular hypertension and glaucoma. Ophthalmology. 1998;105:2077-2081.

3. Mok KH, Lee VW. Nerve fiber analyzer and short-wavelength automated perimetry in glaucoma suspects. A pilot study. Ophthalmology. 2000;107: 2101-2104.

4. Yamazaki Y, Mizuki K, Hayamizu F, Tanaka C. Correlation of blue chromatic macular sensitivity with optic disc change in early glaucoma patients. Jpn J Ophthalmol. 2002;46:89-94. 
5. Arvind $\mathrm{H}$, Graham S, Leaney J, et al. Identifying preperimetric functional loss in glaucoma. A blue-on-yellow multifocal visual evoked potentials study. Ophthalmology. 2009;116:1134-1141.

6. Zhong Y, Zhou X, Cheng Y, Xie L. Relation between blue-on-yellow perimetry and optical coherence tomography in normal tension glaucoma. Can J Ophthalmol. 2010;45:494-500.

7. Remky A, Elsner AE. Blue on yellow perimetry with scanning laser ophthalmoscopy in patients with age related macular disease. $\mathrm{Br} J$ Ophthalmol. 2005;89:464-469.

8. Terasaki H, Miyake Y, Nomura R, Horiguchi M, Suzuki S, Kondo M. Blue-on-yellow perimetry in the complete type of congenital stationary night blindness. Invest Ophthalmol Vis Sci. 1999;40:2761-2764.

9. Machida S, Haga-Sano T, Kizawa J, Tazawa Y. Decrease of blue cone sensitivity in acute idiopathic blind spot enlargement syndrome. Am J Ophthalmol. 2004;138:296-299.

10. Keltner JL, Johnson CA. Short-wavelength automated perimetry in neuroophthalmologic disorders. Arch Ophthalmol. 1995;113:475-481.

11. Wakai M, Takada R, Suzuki K, Trakagi M, Hasegawa S, Abe H. Comparison of Humphrey white-in-white and blue-on-yellow perimetry in monitoring recovery from optic neuropathy. Folia Ophthalmol Jpn. 1997;48:1319-1323.
12. Walters JW, Gaume A, Pate L. Short wavelength-automated perimetry compared with standard achromatic perimetry in autosomal dominant optic atrophy. Br J Ophthalmol. 2006;90:1267-1270.

13. Fujimoto N, Adachi-Usami E. Use of blue-on-yellow perimetry to demonstrate quadrantanopsia in multiple sclerosis. Arch Ophthalmol. 1998;116:828-829.

14. Fujimoto N, Kubota M, Saeki N, Adachi-Usami E. Use of blue-on-yellow perimetry for detection of sectoranopia. Eye. 2004;18:338-341.

15. Kim YY, Kim JS, Shin DH, Kim C, Jung HR. Effect of cataract extraction on blue-on-yellow visual field. Am J Ophthalmol. 2001;132:217-220.

16. Kwon YH, Park HJ, Jap A, Ugurlu S, Caprioli J. Test-retest variability of blue-on-yellow perimetry is greater than white-on-white perimetry in normal subjects. Am J Ophthalmol. 1998;126:29-36.

17. Ito Y, Nakamura M, Yamakoshi T, Lin J, Yatsuya H, Terasaki H. Reduction of inner retinal thickness in patients with autosomal dominant optic atrophy associated with OPA1 mutations. Invest Ophthalmol Vis Sci. 2007;48:4079-4086.

18. Takasugi M, Hayashi T, Okude S, et al. Assessment of macular function in dominant optic atrophy associated with mutation in the OPA1 gene. Jpn Orthopt J. 2010;39:117-122.
Clinical Ophthalmology

\section{Publish your work in this journal}

Clinical Ophthalmology is an international, peer-reviewed journal covering all subspecialties within ophthalmology. Key topics include: Optometry; Visual science; Pharmacology and drug therapy in eye diseases; Basic Sciences; Primary and Secondary eye care; Patien Safety and Quality of Care Improvements. This journal is indexed on

Submit your manuscript here: http://www.dovepress.com/clinical-ophthalmology-journal

\section{Dovepress}

PubMed Central and CAS, and is the official journal of The Society of Clinical Ophthalmology (SCO). The manuscript management system is completely online and includes a very quick and fair peer-review system, which is all easy to use. Visit http://www.dovepress.com/ testimonials.php to read real quotes from published authors. 